\title{
INEQUALITIES FOR DUAL AFFINE QUERMASSINTEGRALS
}

\author{
YUAN JUN AND LENG GANGSONG
}

Received 18 April 2005; Revised 2 November 2005; Accepted 8 November 2005

For star bodies, the dual affine quermassintegrals were introduced and studied in several papers. The aim of this paper is to study them further. In this paper, some inequalities for dual affine quermassintegrals are established, such as the Minkowski inequality, the dual Brunn-Minkowski inequality, and the Blaschke-Santaló inequality.

Copyright (c) 2006 Y. Jun and L. Gangsong. This is an open access article distributed under the Creative Commons Attribution License, which permits unrestricted use, distribution, and reproduction in any medium, provided the original work is properly cited.

\section{Introduction}

The setting for this paper is $n$-dimensional Euclidean space $\mathbb{R}^{n}$. Let $\mathscr{K}^{n}$ denote the set of convex bodies (compact, convex subsets with nonempty interiors) and $\mathcal{Y}_{o}^{n}$ denote the subset of $\mathscr{K}^{n}$ that consists of convex bodies with the origin in their interiors. Denote by $\operatorname{vol}_{i}(K \mid \xi)$ the $i$-dimensional volume of the orthogonal projection of $K$ onto an $i$ dimensional subspace $\xi \subset \mathbb{R}^{n}$. Affine quermassintegrals are important geometric invariants related to the projection of convex body. These quermassintegrals were introduced by Lutwak [7], and can be defined by letting $\Phi_{0}(K)=V(K), \Phi_{n}(K)=k_{n}$, and for $0<i<n$,

$$
\Phi_{i}(K)=k_{n}\left(\int_{G(n, n-i)}\left[\frac{\operatorname{vol}_{n-i}(K \mid \xi)}{k_{n-i}}\right]^{-n} d \xi\right)^{-1 / n}
$$

where the Grassmann manifold $G(n, i)$ is endowed with the normalized Haar measure, and $k_{n}$ is the volume of the unit ball $B_{n}$ in $\mathbb{R}^{n}$.

Furthermore, in [6], Lutwak introduced the dual affine quermassintegrals of a star body $L$ containing the origin in its interior, $\widetilde{\Phi}_{i}(L)$, by letting $\widetilde{\Phi}_{0}(L)=V(L), \widetilde{\Phi}_{n}(L)=k_{n}$, and for $0<i<n$,

$$
\widetilde{\Phi}_{i}(L)=k_{n}\left(\int_{G(n, n-i)}\left[\frac{\operatorname{vol}_{n-i}(L \cap \xi)}{k_{n-i}}\right]^{n} d \xi\right)^{1 / n}
$$

Hindawi Publishing Corporation Journal of Inequalities and Applications Volume 2006, Article ID 50181, Pages 1-7 DOI 10.1155/JIA/2006/50181 
where $\operatorname{vol}_{i}(L \cap \xi)$ denotes the $i$-dimensional volume of intersection of $L$ with an $i$-dimensional subspace $\xi \subset \mathbb{R}^{n}$.

Grinberg [4] proved that both the affine quermassintegrals and the dual affine quermassintegrals are invariant under volume-preserving affine transformations.

For star bodies, the dual affine quermassintegrals were studied in $[3,4,7,10]$. The aim of this paper is to study them further. For reader's convenience, we try to make the paper self-contained. This paper, except for the introduction, is divided into three sections. In Section 2 we recall some basics about convex bodies, star bodies, and dual mixed volume.

In Section 3, we introduce the concept of the mixed p-dual affine quermassintegrals and establish the Minkowski inequality for them (Theorem 3.1). As an application, the dual Brunn-Minkowski inequality for the dual affine quermassintegrals is obtained.

In Section 4, we establish a connection between the affine quermassintegrals and the dual affine quermassintegrals for a given convex body.

\section{Notation and preliminary works}

As usual, $S^{n-1}$ denotes the unit sphere, $B_{n}$ the unit ball, and $o$ the origin in Euclidean $n$-space $\mathbb{R}^{n}$.

Let $K$ be a nonempty compact convex body in $\mathbb{R}^{n}$, the support function $h_{K}$ of $K$ is defined by

$$
h_{K}(u)=\max \{u \cdot x: x \in K\}, \quad u \in S^{n-1},
$$

where $u \cdot x$ denotes the usual inner product of $u$ and $x$ in $\mathbb{R}^{n}$.

If $K$ is a convex body that contains the origin in its interior, the polar body $K^{*}$ of $K$, with respect to the origin, is defined by

$$
K^{*}=\left\{x \in \mathbb{R}^{n} \mid x \cdot y \leq 1, y \in K\right\} .
$$

For a compact subset $L$ of $\mathbb{R}^{n}$, which is star-shaped with respect to the origin, we will use $\rho(L, \cdot)$ to denote its radial function; that is, for $u \in S^{n-1}$,

$$
\rho(L, u)=\rho_{L}(u)=\max \{\lambda>0: \lambda u \in L\}
$$

If $\rho(L, \cdot)$ is continuous and positive, $L$ will be called a star body.

Let $\mathscr{S}_{o}^{n}$ denote the set of star bodies in $\mathbb{R}^{n}$ containing the origin in their interiors. Two star bodies $K, L \in \mathscr{S}_{o}^{n}$ are said to be dilatate (of one another) if $\rho(K, u) / \rho(L, u)$ is independent of $u \in S^{n-1}$.

Let $L_{j} \in \mathscr{Y}_{o}^{n}(1 \leq j \leq n)$. The dual mixed volume $\tilde{V}\left(L_{1}, \ldots, L_{n}\right)$ is defined by

$$
\tilde{V}\left(L_{1}, \ldots, L_{n}\right)=\frac{1}{n} \int_{S^{n-1}} \rho_{L_{1}}(u) \rho_{L_{2}}(u) \cdots \rho_{L_{n}}(u) d u .
$$

We use the notation $\tilde{V}\left(L_{1}, i_{1} ; \ldots ; L_{n}, i_{n}\right)$ for the dual mixed volume in which $L_{j}$ appears $i_{j}$ times.

If $x_{i} \in \mathbb{R}^{n}, 1 \leq i \leq m$, then $x_{1} \tilde{+} \cdots \tilde{+} x_{m}$ is defined to be the usual vector sum of the points $x_{i}$, if all of them belong to a line through $o$, and 0 otherwise. 
Let $L_{i} \in \mathscr{Y}_{o}^{n}$ and $t_{i} \geq 0,1 \leq i \leq m$, then

$$
t_{1} L_{1} \tilde{+} \cdots \tilde{+} t_{m} L_{m}=\left\{t_{1} x_{1} \tilde{+} \cdots \tilde{+} t_{m} x_{m}: x_{i} \in L_{i}\right\}
$$

is called a radial linear combination.

The following elementary property of dual mixed volumes will be used later. For $K, L, L_{j} \in \mathscr{Y}_{o}^{n}(1 \leq j \leq n-1)$,

$$
\tilde{V}\left(L_{1}, \ldots, L_{n-1}, K \tilde{+} L\right)=\tilde{V}\left(L_{1}, \ldots, L_{n-1}, K\right)+\tilde{V}\left(L_{1}, \ldots, L_{n-1}, L\right)
$$

For $K, L \in \mathscr{Y}_{o}^{n}$, the Minkowski inequality for dual mixed volumes [3, page 373] states

$$
\tilde{V}(K, n-p ; L, p)^{n} \leq V(K)^{n-p} V(L)^{p},
$$

with equality if and only if $K$ is a dilatate of $L$.

The above elementary results (and definitions) are from the theory of convex bodies. The reader may consult the standard works on the subject $[1,3,5,9,10]$ for reference.

\section{The dual Brunn-Minkowski inequalities for dual affine quermassintegrals}

In this section, we will prove the dual Brunn-Minkowski inequality for the dual harmonic quermassintegrals. At first, we introduce the concept of mixed $p$-dual affine quermassintegrals.

Let $K, L \in \mathscr{Y}_{o}^{n}, \xi \in G(n, i)$ and $0 \leq p \leq i$. We define mixed $p$-dual affine quermassintegrals, $\widetilde{\Phi}_{p, i}(K, L)$. Let first $\widetilde{V}_{p, i}(K, L ; \xi)$ by

$$
\tilde{V}_{p, i}(K, L ; \xi)=\tilde{V}(K \cap \xi, i-p ; L \cap \xi, p) .
$$

It is easy to verify that $\tilde{V}_{p, i}(K, K ; \xi)=\operatorname{vol}_{i}(K \cap \xi)$, for all $0 \leq p \leq n-i$, and $\tilde{V}_{i, i}(K, L)=$ $\operatorname{vol}_{i}(L \cap \xi)$, for all $K$.

Now we define the mixed $p$-dual affine quermassintegrals $\widetilde{\Phi}_{p, i}(K, L)$ by

$$
\widetilde{\Phi}_{p, i}(K, L)=k_{n}\left(\int_{G(n, n-i)}\left[\frac{\tilde{V}_{p, n-i}(K, L ; \xi)}{k_{n-i}}\right]^{n} d \xi\right)^{1 / n}
$$

If $p=1$, we will write $\widetilde{\Phi}_{i}(K, L)$, rather than $\widetilde{\Phi}_{1, i}(K, L)$. It follows that $\widetilde{\Phi}_{p, i}(K, K)=\widetilde{\Phi}_{i}(K)$, for all $0 \leq p \leq n-i$ and $\widetilde{\Phi}_{n-i, i}(K, L)=\widetilde{\Phi}_{i}(L)$, for all $K$.

For the mixed $p$-dual affine quermassintegrals, we have the following Minkowski inequality.

Theorem 3.1. Let $K, L \in \mathscr{Y}_{o}^{n}$ and $0 \leq i<n$. If $0 \leq p \leq i$, then

$$
\tilde{\Phi}_{p, i}(K, L)^{n-i} \leq \tilde{\Phi}_{i}(K)^{n-i-p} \tilde{\Phi}_{i}(L)^{p},
$$

with equality if and only if $K$ is a dilatate of $L$. 
4 Inequalities for dual affine quermassintegrals

Proof. Let $\xi \in G(n, n-i)$. By (2.7), we get

$$
\begin{aligned}
\tilde{V}_{p, n-i}(K, L ; \xi) & =\tilde{V}(K \cap \xi, n-i-p ; L \cap \xi, p) \\
& \leq \operatorname{vol}_{n-i}(K \cap \xi)^{(n-i-p) /(n-i)} \operatorname{vol}_{n-i}(L \cap \xi)^{p /(n-i)} .
\end{aligned}
$$

According to (3.4) and the Hölder integral inequality, we have

$$
\begin{aligned}
\widetilde{\Phi}_{p, i}(K, L) & =k_{n}\left(\int_{G(n, n-i)}\left[\frac{\tilde{V}_{p, n-i}(K, L ; \xi)}{k_{n-i}}\right]^{n} d \mu_{i}(\xi)\right)^{1 / n} \\
& \leq k_{n}\left(\int_{G(n, n-i)}\left[\frac{\operatorname{vol}_{n-i}(K \cap \xi)}{k_{n-i}}\right]^{n(n-i-p) /(n-i)}\left[\frac{\operatorname{vol}_{n-i}(L \cap \xi)}{k_{n-i}}\right]^{n p /(n-i)} d \mu_{i}(\xi)\right)^{1 / n} \\
& \leq \widetilde{\Phi}_{i}(K)^{(n-i-p) /(n-i)} \tilde{\Phi}_{i}(L)^{p /(n-i)}
\end{aligned}
$$

By the equality conditions of Hölder integral inequality and the Minkowski inequality for dual mixed volumes, the equality of (3.3) holds if and only if $K$ is a dilatate of $L$.

As an application of Theorem 3.1, we have the following dual Brunn-Minkowski inequality for the dual affine quermassintegrals.

Theorem 3.2. Let $K, L \in \mathscr{Y}_{o}^{n}$ and $0 \leq i \leq n-1$. Then

$$
\widetilde{\Phi}_{i}(K \widetilde{+} L)^{1 /(n-i)} \leq \widetilde{\Phi}_{i}(K)^{1 /(n-i)}+\widetilde{\Phi}_{i}(L)^{1 /(n-i)},
$$

with equality if and only if $K$ is a dilatate of $L$.

Proof. Let $\xi \in G(n, i)$ and $K, L \in \mathscr{Y}_{o}^{n}$, it is easy to prove that

$$
(K \tilde{+} L) \cap \xi=(K \cap \xi) \widetilde{+}(L \cap \xi) .
$$

In fact, for $u \in S^{n-1} \cap \xi$, we have

$$
\rho_{(K \tilde{+} L) \cap \xi}(u)=\rho_{K \tilde{+} L}(u)=\rho_{K}(u)+\rho_{L}(u)=\rho_{K \cap \xi}(u)+\rho_{L \cap \xi}(u)=\rho_{K \cap \xi \tilde{+} L \cap \xi}(u) .
$$

By (2.6), (3.7), for $M \in \mathscr{Y}_{o}^{n}$, we have

$$
\begin{aligned}
\tilde{V}_{1, i}(M, K \tilde{+} L ; \xi) & =\tilde{V}(M \cap \xi, i-1 ;(K \tilde{+} L) \cap \xi) \\
& =\widetilde{V}(M \cap \xi, i-1 ;(K \cap \xi) \tilde{+}(L \cap \xi)) \\
& =\widetilde{V}(M \cap \xi, i-1 ; K \cap \xi)+\tilde{V}(M \cap \xi, i-1 ; L \cap \xi) \\
& =\widetilde{V}_{1, i}(M, K ; \xi)+\tilde{V}_{1, i}(M, L ; \xi) .
\end{aligned}
$$


According to (3.2) and Minkowski integral inequality, we have

$$
\begin{aligned}
\widetilde{\Phi}_{i}(M, K \tilde{+} L) & =k_{n}\left(\int_{G(n, n-i)}\left[\frac{\tilde{V}_{1, n-i}(M, K \tilde{+} L ; \xi)}{k_{n-i}}\right]^{n} d \mu_{n-i}(\xi)\right)^{1 / n} \\
& =k_{n}\left(\int_{G(n, n-i)}\left[\frac{\tilde{V}_{1, n-i}(M, K ; \xi)+\tilde{V}_{1, n-i}(M, L ; \xi)}{k_{n-i}}\right]^{n} d \mu_{n-i}(\xi)\right)^{1 / n} \\
& \leq \widetilde{\Phi}_{i}(M, K)+\widetilde{\Phi}_{i}(M, L) \leq \widetilde{\Phi}_{i}(M)^{(n-i-1) /(n-i)}\left(\widetilde{\Phi}_{i}(K)^{1 /(n-i)}+\widetilde{\Phi}_{i}(L)^{1 /(n-i)}\right),
\end{aligned}
$$

with equality if and only if $K$ and $L$ are dilatate of $M$. Now we take $K \tilde{+} L$ for $M$, and recall that $\widetilde{\Phi}_{i}(K, K)=\widetilde{\Phi}_{i}(K)$; then Theorem 3.2 follows.

Remark 3.3. Theorem 3.2 is a dual of Lutwak's inequality for affine quermassintegrals, which was proved in [7]: let $K$ and $L$ be convex bodies in $\mathbb{R}^{n}$ and $0 \leq i \leq n-1$, then

$$
\Phi_{i}(K+L)^{1 /(n-i)} \geq \Phi_{i}(K)^{1 /(n-i)}+\Phi_{i}(L)^{1 /(n-i)},
$$

with equality if and only if $K$ and $L$ are homothetic.

\section{More about the dual affine quermassintegrals}

Let $K$ be a convex body of constant width, $K^{*}$ is the polar body of $K$. We proved that among convex bodies of constant width, precisely the ball attains the minimal value of $\tilde{\Phi}_{n-1}\left(K^{*}\right)$.

THEOREM 4.1. Let $K \in \mathcal{Y}_{o}^{n}$. If

$$
\operatorname{vol}_{1}(K \mid \xi)=\operatorname{vol}_{1}\left(B_{n} \mid \xi\right)
$$

for all $\xi \in G(n, 1)$, then

$$
\tilde{\Phi}_{n-1}\left(K^{*}\right) \geq \tilde{\Phi}_{n-1}\left(B_{n}^{*}\right)
$$

with equality if and only if $K=B_{n}$.

Proof. For all $u \in S^{n-1},(4.1)$ is equivalent to

$$
h(K, u)+h(K,-u)=2,
$$

and the chord length of $K^{*}$ in direction $u$ satisfies

$$
\rho\left(K^{*}, u\right)+\rho\left(K^{*},-u\right) \geq \frac{4}{h(K, u)+h(K,-u)}=2,
$$

where we have used the inequality between arthmetic and harmonic means. 
6 Inequalities for dual affine quermassintegrals

Notice that if $\xi \in G(n, 1)$, then $\operatorname{vol}_{1}\left(K^{*} \cap \xi\right)$ is just the chord length of $K^{*}$ along $\xi$. By (1.2), we have

$$
\begin{aligned}
\tilde{\Phi}_{n-1}\left(K^{*}\right) & =k_{n}\left(\int_{G(n, 1)}\left[\frac{\operatorname{vol}_{1}\left(K^{*} \cap \xi\right)}{2}\right]^{n} d \xi\right)^{1 / n} \\
& =k_{n}\left(\frac{1}{n k_{n}} \int_{S^{n-1}}\left[\frac{\rho\left(K^{*}, u\right)+\rho\left(K^{*},-u\right)}{2}\right]^{n} d u\right)^{1 / n} \geq k_{n}=\widetilde{\Phi}_{n-1}\left(B_{n}^{*}\right) .
\end{aligned}
$$

Equality holds if and only if $h(K, u)=h(K,-u)=1$, which implies $K$ is a unit ball centered at the origin.

The following theorem which establishes a connection between the affine quermassintegrals and the dual affin equermassintegrals generalizes the Blaschke-Santaló inequality. Theorem 4.2. Let $K$ be a centered convex body and $0 \leq i<n$. Then

$$
\tilde{\Phi}_{i}\left(K^{*}\right) \Phi_{i}(K) \leq k_{n}^{2}
$$

with equality if and only if $K$ is an ellipsoid.

To prove the inequality (4.6), the following lemma will be needed.

Lemma $4.3[8]$. Let $K \in \mathcal{Y}_{o}^{n}$ and $\xi \in G(n, i)$. Then

$$
K^{*} \cap \xi=(K \mid \xi)^{*}
$$

Proof of Theorem 4.2. Let $s=n-i$, and $\xi \in G(n, s)$. By the Blaschke-Santaló inequality, for the body $K \mid \xi$ in $\xi$, we have

$$
\operatorname{vol}_{s}\left[(K \mid \xi)^{*}\right] \operatorname{vol}_{s}(K \mid \xi) \leq k_{s}^{2}
$$

with equality if and only if $K \mid \xi$ is an ellipsoid in $\xi$.

According to Lemma 4.3, we obtain

$$
\left[\frac{V_{s}\left(K^{*} \cap \xi\right)}{k_{s}}\right]^{n} \leq\left[\frac{V_{s}(K \mid \xi)}{k_{s}}\right]^{-n},
$$

with equality if and only if $K \mid \xi$ is an ellipsoid in $\xi$. We integrate both sides of inequality (4.9) over $G(n, s)$ and get

$$
\left(\frac{\tilde{\Phi}_{i}\left(K^{*}\right)}{k_{n}}\right)^{n} \leq\left(\frac{\Phi_{i}(K)}{k_{n}}\right)^{-n} .
$$

This is the desired inequality

$$
\tilde{\Phi}_{i}\left(K^{*}\right) \Phi_{i}(K) \leq k_{n}^{2}
$$

with equality if and only if $K$ is an ellipsoid. The equality condition follows from the fact that, for $s>1$, ellipsoid is the only body all of whose $s$-dimensional projections are $s$-dimensional ellipsoids (see [3, page 95]). 
Remark 4.4. The case $i=0$ of (4.6) is the well-known Blaschke-Santaló inequality.

\section{Acknowledgments}

The authors are most grateful to the referees for their helpful suggestions. This work was supported in part by the National Natural Science Foundation of China. (Grant no. 10271071)

\section{References}

[1] T. Bonnesen and W. Fenchel, Theorie der konvexen Körper, Springer, Berlin, 1934.

[2] H. Federer, Geometric Measure Theory, Die Grundlehren der mathematischen Wissenschaften, vol. 153, Springer, New York, 1969.

[3] R. J. Gardner, Geometric Tomography, Encyclopedia of Mathematics and Its Applications, vol. 58, Cambridge University Press, Cambridge, 1995.

[4] E. L. Grinberg, Isoperimetric inequalities and identities for $k$-dimensional cross-sections of a convex bodies, London Mathematical Society 22 (1990), 478-484.

[5] K. Leichtweiss, Konvexe Mengen, Springer, Berlin, 1980.

[6] E. Lutwak, Dual mixed volumes, Pacific Journal of Mathematics 58 (1975), no. 2, 531-538.

[7] _ A general isepiphanic inequality, Proceedings of the American Mathematical Society 90 (1984), no. 3, 415-421.

[8] _ Inequalities for Hadwiger's harmonic quermassintegrals, Mathematische Annalen 280 (1988), no. 1, 165-175.

[9] L. A. Santaló, Integral Geometry and Geometric Probability, Encyclopedia of Mathematics and Its Applications, vol. 1, Addison-Wesley, Massachusetts, 1976.

[10] R. Schneider, Convex Bodies: the Brunn-Minkowski Theory, Encyclopedia of Mathematics and Its Applications, vol. 44, Cambridge University Press, Cambridge, 1993.

Yuan Jun: Department of Mathematics, Shanghai University, Shanghai 200444, China

E-mail address: yuanjun@graduate.shu.edu.cn

Leng Gangsong: Department of Mathematics, Shanghai University, Shanghai 200444, China

E-mail address: gleng@staff.shu.edu.cn 